\title{
Unique epigenetic gene profiles define human breast cancers with poor prognosis
}

\author{
Samuel Peña-Llopis ${ }^{1,2}$, Yihong Wan ${ }^{1}$ and Elisabeth D. Martinez ${ }^{1}$ \\ 1 Department of Pharmacology, UT Southwestern Medical Center, Dallas, TX, USA \\ ${ }^{2}$ Department of Translational Oncology, National Center for Tumor Diseases and German Cancer Research Center, Heidelberg, \\ Germany \\ Correspondence to: Samuel Peña-Llopis, email: Samuel.Pena.Llopis@gmail.com \\ Elisabeth D. Martinez, email: elisabeth.martinez@utsouthwestern.edu \\ Keywords: epigenetic signature, expression profiles, prognosis, molecular targets, triple negative breast cancer \\ Received: October 24, $2016 \quad$ Accepted: October 29, $2016 \quad$ Published: November 14, 2016
}

\section{ABSTRACT}

Epigenetic enzymes are at the nexus of cellular regulatory cascades and can drive cancer-specific deregulation at all stages of the oncogenic process, yet little is known about their prognostic value in human patients. Here, we used qRT-PCR to profile at high resolution the expression of fifty-five epigenetic genes in over one hundred human breast cancer samples and patient-matched benign tissues. We correlated expression patterns with clinical and histological parameters and validated our findings in two independent large patient cohorts (TCGA and METABRIC). We found that human breast malignancies have unique epigenetic profiles and cluster into epigenetic subgroups. A subset of epigenetic genes defined an Epigenetic Signature as an independent predictor of patient survival that outperforms triple negative status and other clinical variables. Our results also suggest that breast cancer grade, but not stage, is driven by transcriptional alterations of epigenetic modifiers. Overall, this study uncovers the presence of epigenetic subtypes within human mammary malignancies and identifies tumor subgroups with specific pharmacologically targetable epigenetic susceptibilities not yet therapeutically exploited.

\section{INTRODUCTION}

It has been estimated that epigenetic changes are ten to forty times more frequent in cancers, including breast cancer, than genetic mutations [1-4]. Recent reports have described the over-expression, amplification, fusion or mutation of many individual epigenetic enzymes across a variety of tumor types. Epigenetic enzymes have the potential to influence cellular pathways beyond control of chromatin structure [1-4], affecting the modulation of transcription factor function $[5,6]$ and of protein synthesis [7] and stability [8]. These facts put epigenetic enzymes at the nexus of cellular regulatory cascades and define them as potential drivers of cancer-specific deregulation at all stages of the oncogenic process.

Individual epigenetic genes have been found to be oncogenic drivers and thus therapeutic targets [9-11] or to contribute to the oncogenic process through loss of function, or new mutant activities [12,13]. The epigenetic landscape impacts breast cancer susceptibility and affects metabolic status, oncogene addiction, tumor suppressor silencing and even the development of drug resistance [1-4, 14-19]. Here, we used quantitative high throughput RT-PCR to measure the expression of 55 epigenetic genes in over 100 fully annotated breast cancer patient samples and the corresponding patient-matched benign specimens. We then validated our results using The Cancer Genome Atlas (TCGA) data (https://tcga-data.nci.nih.gov/tcga) and, separately, using the Molecular Taxonomy of Breast Cancer International Consortium (METABRIC) dataset [20]. We found that levels of epigenetic genes distinguish normal vs. malignant tissues, that epigenetic subtypes exist within human breast cancers, and in particular, that a unique epigenetic gene signature has stronger prognostic value than triple negative status, and identifies enzymes that can be pharmacologically targeted, suggesting novel therapeutic options for human patients. 


\section{RESULTS}

\section{Human breast cancers exhibit altered epigenetic gene expression profiles}

We obtained over 100 tumor specimens (and more than 80 corresponding patient-matched benign tissues) through UTSTR core facility (Supplementary Table 1) scored pathologically to contain $>70 \%$ tumor tissue and otherwise randomly selected [21]. We performed qRT-PCR analysis in triplicate to measure the expression level of 55 detectable epigenetic enzyme genes (Supplementary Table 2 ), plus three reference and three tissue specific control genes. Expression profiles of the 55 genes were sufficient to cluster tumor samples away from benign tissue (Figure 1 and Supplementary Figure 1). No lobular breast cancers clustered with benign tissues although a few ductal samples did. Among the changes driving this benign $v s$. tumor separation (Figure 1 and Supplementary Figure 1A), we observed the downregulation of HDAC 3 and Sirt2 as

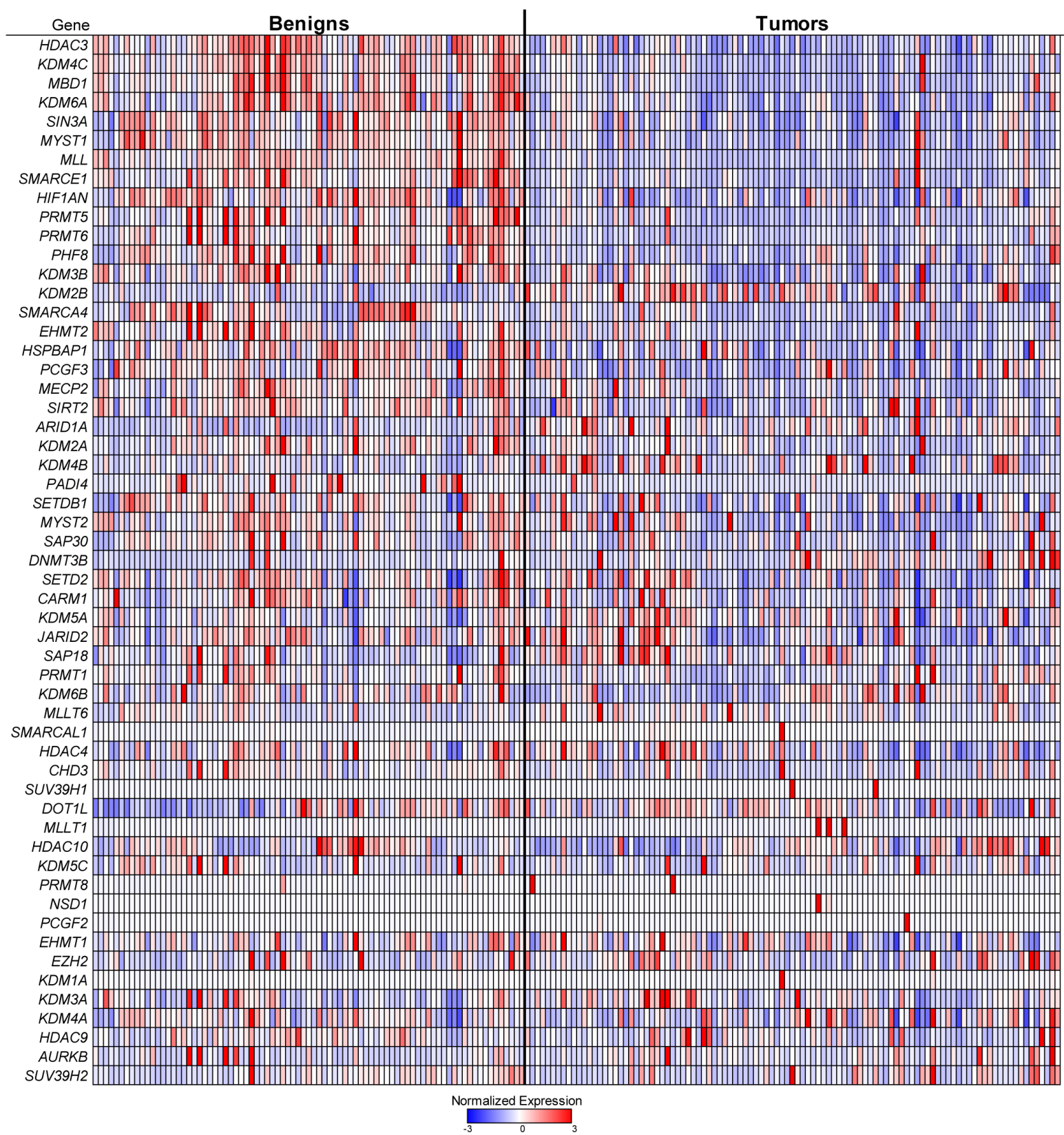

p FDR $q$ FC 7E-15 4E-13 -1.5 6E-13 2E-11 -1.9 $3 \mathrm{E}-12$ 5E-11 -2.0 4E-12 5E-11 -1.7 1E-11 1E-10 -1.5 6E-10 5E-9 -1.6 2E-9 1E-8 -2.0 2E-9 1E-8 -2.0 $\begin{array}{lll}5 \mathrm{E}-9 & 3 \mathrm{E}-8 & -1.5\end{array}$ 2E-8 9E-8 -1.8 $\begin{array}{lll}3 E-8 & 1 E-7 & -2.3\end{array}$ 3E-8 1E-7 -1.6 1E-7 5E-7 -1.4 $1 E-7 \quad 5 E-7 \quad 1.6$ 1E-7 5E-7 -1.5 3E-5 1E-4 -1.4 5E-5 2E-4 - 1.3 6E-5 2E-4 -1.4 7E-5 2E-4 -1.4 7E-5 2E-4 -1.3 2E-4 5E-4 1.6 4E-4 9E-4 -1.5 4E-4 1E-3 1.5 $\begin{array}{lll}7 E-4 & 0.002 & -5.1\end{array}$ $\begin{array}{llll}7 \mathrm{7E}-4 & \mathbf{0 . 0 0 2} & -1.2\end{array}$ $\begin{array}{llll}1 E-3 & 0.002 & -1.3\end{array}$ $\begin{array}{llll}0.002 & 0.005 & -1.4\end{array}$ $\begin{array}{lll}0.005 & 0.009 & 1.6\end{array}$ $\begin{array}{lll}\mathbf{0 . 0 0 5} & \mathbf{0 . 0 0 9} & -1.2\end{array}$ $\begin{array}{llll}\mathbf{0 . 0 0 5} & \mathbf{0 . 0 0 9} & -1.2\end{array}$ $\begin{array}{lll}0.021 & 0.038 & 1.2\end{array}$ $\begin{array}{lll}\mathbf{0 . 0 2 7} & \mathbf{0 . 0 4 6} & -1.1\end{array}$ $\begin{array}{lll}\mathbf{0 . 0 4 2} & 0.07 & 1.2\end{array}$ $\begin{array}{lll}0.045 & 0.07 & -1.2\end{array}$ $\begin{array}{lll}0.08 & 0.12 & -1.2\end{array}$ $\begin{array}{lll}0.09 & 0.14 & 1.2\end{array}$ $\begin{array}{lll}0.11 & 0.16 & 2.3\end{array}$

Figure 1: Distinct patterns of epigenetic gene expression in patient-matched benign vs. tumor tissues. A., Epigenetic genes were measured by qRT-PCR. For each gene, the $p$ value, false discovery rate (FDR) $q$ value, and fold change (FC) are given across all tumor $v s$. benign samples ( $n=103$ and 83, respectively). Expression values were categorized by the number of standard deviations away from the average $(-3$ to +3$)$ for each gene across all samples and are represented in a blue to red color scale. See also Supplementary Figure 1 . 
reported [22, 23]. In addition, we found altered expression of subsets of all major histone erasers, writers, readers and modifiers. For instance, among erasers, we saw general cancer downregulation of KDM4C/JMJD2C (negatively associated with invasive breast cancer [24]). In contrast, $K D M 2 B / F B X L 10$ and $K D M 4 B / J M J D 2 B$ were upregulated while $K D M 3 A / J M J D 1 A, K M D 2 A / J M J D 2 A$ and $K D M 5 C /$ $J A R I D 1 C$ were not altered. Among histone writers, $M L L$, EHMT2, PRMT5 and PRMT6 were downregulated with no changes in DOT1L and MLLT6. Of note was the increased expression of $A R I D 1 A$ (perhaps due to mutations that decrease protein levels [25]), and of DNMT3B. Patientmatched benign $v s$. tumor paired analysis also revealed inter-patient heterogeneity (Supplementary Figure 1B). These results indicate that epigenetic enzyme genes are generally deregulated in breast tumors.

\section{Transcriptional co-regulation of subsets of epigenetic genes in patient samples}

Unsupervised hierarchical clustering grouped genes into four major subsets in tumors (Supplementary Figure 2A). Jumonji enzymes are over-represented in one subset, for example, while several histone methylases cluster together with $A U R K B$. The gene to gene correlation profiles in benign tissue followed a distinct though partly overlapping pattern compared to tumor samples (Supplementary Figure 2B). The histone methylase/AURKB cluster, for example, is not present in benign tissue. Most striking were the large distances seen between PAD14, HDAC10 or DOT1L to other genes in the benign tissue dataset, with only PADI4 remaining isolated in the tumor tissue, suggestive of new tumorigenic transcriptional networks involving $H D A C 10$ and independently, DOT1L.

\section{Epigenetic gene levels define breast cancer subgroups}

Upon examination of correlations between expression levels and clinical information, several new findings became evident (Figure 2A and Supplementary Figure 3). We found, for example, that even after strict false discovery rate (FDR) correction, EZH2 and $A U R K B$ levels showed robust positive correlation with triple negative (TN) malignancies and high $\mathrm{Ki} 67$ index, as expected from their role in tumor aggressiveness (Supplementary Figure 3A). Levels of EZH2 and AURKB increased with tumor grade and positively correlated with p53 levels. Several novel genes also positively correlated with TN disease, high Ki67 levels and tumor grade: DNMT3B, SUV39H1 and SUV39H2 (Figure 2A and Supplementary Figure 3A). Levels of several epigenetic genes negatively correlated with TN disease including KDM4B/JMJD2B, MYST1 and MYST2, PRMT8 and
SIN3A. Of these, KDM4B/JMJD2B, MYST1, MYST2 and $P R M T 8$ were specifically upregulated in grade 2 tumors, while $\operatorname{SIN} 3 A$ was downregulated in grade 3 malignancies. Additionally, CHD3 levels negatively correlated with tumor grade, and PCGF2 and PCGF3 levels positively correlated with ER+ status (Figure 2A). Altogether, twelve genes showed strong correlations with tumor grade/receptor status after stringent FDR corrections (Figure 2A), which hereafter we denominate "Epigenetic Signature". These correlations were all lacking in benign tissue (Supplementary Figure 3B), indicating that breast cancers may fall into functional epigenetic subgroups.

To validate these findings, we analyzed the twelve genes in the Epigenetic Signature derived from our qRTPCR UTSW cohort, in the BRCA-TCGA RNA-Seq as well as the METABRIC datasets [20] that had become publicly available. In the BRCA-TCGA set, we found that with the exception of PRMT8, for which only a few measurements were available, the remaining eleven genes showed highly significant correlations with TN tumors (Figure 2B), confirming our UTSW results. Similarly, the METABRIC dataset validated the correlation of ten of the twelve genes with TN status and additionally confirmed the correlation of nine of the Epigenetic Signature genes with tumor grade (Supplementary Figure 4A). Note that grade information is not readily available on the TCGA portal. The expression levels of the eleven validated genes (common to UTSW and either TCGA or METABRIC, or both) were sufficient to cluster tumors into therapeutically relevant subtypes including grade for UTSW and METABRIC, and TN status for UTSW, BRCA-TCGA and METABRIC (Figures 2C and 2D and Supplementary Figure 4B). Randomly selected groups of 11 genes out of 20,534 transcripts of the RNA-Seq data did not robustly segregate tumors into relevant subgroups (Supplementary Figure 4C), and neither did groups of 11 genes from the measured 55 epigenetic genes after excluding the Epigenetic Signature (Supplementary Figure 4D). To quantify the relative robustness of the separation seen with the Epigenetic Signature genes, we calculated arbitrary distances between the main cluster of normal samples (present in all cases) and the remaining samples, in TCGA. A one-sample $t$-test was performed to compare the distance of the eleven epigenetic genes $v s$. the other sets of random genes. As shown in Supplementary Figures $4 \mathrm{C}-4 \mathrm{D}$, the 11 genes had a distance equal to 0 which was significantly different than the other gene sets, which gave distances ranging from 65-135 $(P=0.044$, Supplementary Figure $4 \mathrm{C} ; P=0.00002$ for comparison to all nonsignature gene sets tested, Supplementary Figure 4C-4D).

We next examined each of the eleven genes (AURKB, DNMT3B, EZH2, SUV39H1, SUV39H2, CHD3, KDM4B, MYST1, PCGF2, PCGF3 and SIN3A) in more detail in the three datasets, and generally found significant differences in their expression in TN vs. nonTN disease (Figure 3). These differences generally also held 
A

\begin{tabular}{lcccccc}
\multirow{2}{*}{ FDR $\boldsymbol{q}$} & \multicolumn{7}{c}{ UTSW } \\
& Grade Stage & ER & PR & HER2 & TN \\
\cline { 2 - 7 } Gene / Test & Correl. & Correl. & $t$ Test & $t$ Test & $t$ Test & $t$ Test \\
\hline AURKB & $\mathbf{8 E}-11$ & 0.99 & $\mathbf{9 E}-\mathbf{5}$ & $\mathbf{0 . 0 0 2}$ & 0.74 & $\mathbf{3 E}-\mathbf{6}$ \\
DNMT3B & $\mathbf{0 . 0 2 5}$ & 0.74 & $\mathbf{0 . 0 4 5}$ & $\mathbf{0 . 0 1 7}$ & 0.74 & $\mathbf{0 . 0 4 3}$ \\
EZH2 & $\mathbf{9 E}-5$ & 0.99 & $\mathbf{0 . 0 2 0}$ & 0.09 & 0.74 & $\mathbf{1 E}-\mathbf{4}$ \\
SUV39H1 & $\mathbf{0 . 0 0 8}$ & 0.99 & $\mathbf{0 . 0 2 0}$ & 0.14 & 0.74 & $\mathbf{0 . 0 0 2}$ \\
SUV39H2 & $\mathbf{1 E - 7}$ & 0.99 & $\mathbf{9 E}-\mathbf{5}$ & $\mathbf{0 . 0 0 5}$ & 0.74 & $\mathbf{6 E}-\mathbf{8}$ \\
CHD3 & $\mathbf{1 E}-\mathbf{3}$ & 0.99 & 0.12 & 0.30 & 0.75 & 0.07 \\
KDM4B & $\mathbf{9 E}-\mathbf{5}$ & 0.99 & $\mathbf{5 E}-5$ & $\mathbf{0 . 0 2 0}$ & 0.81 & $\mathbf{3 E}-\mathbf{4}$ \\
MYST1 & $\mathbf{0 . 0 0 8}$ & 0.99 & $\mathbf{0 . 0 3 5}$ & 0.25 & 0.74 & $\mathbf{0 . 0 0 3}$ \\
PCGF2 & $\mathbf{0 . 0 0 8}$ & 0.99 & $\mathbf{0 . 0 2 9}$ & 0.30 & 0.80 & 0.08 \\
PCGF3 & $\mathbf{0 . 0 1 4}$ & 0.99 & $\mathbf{0 . 0 2 5}$ & 0.32 & 0.80 & 0.07 \\
PRMT8 & $\mathbf{0 . 0 1 9}$ & 0.60 & $\mathbf{9 E}-\mathbf{4}$ & $\mathbf{0 . 0 1 6}$ & 0.74 & $\mathbf{4 E - 4}$ \\
SIN3A & $\mathbf{0 . 0 0 8}$ & 0.99 & $\mathbf{0 . 0 0 9}$ & 0.14 & 0.98 & $\mathbf{0 . 0 3 2}$
\end{tabular}

B

\begin{tabular}{|c|c|c|c|c|c|c|c|c|}
\hline \multirow{2}{*}{ FDR $q$} & \multicolumn{7}{|c|}{ BRCA-TCGA } & \multirow[b]{2}{*}{ TN } \\
\hline & pT & $\mathrm{pN}$ & pM & Stage & ER & PR & HER2 & \\
\hline Gene / Test & Correl. & $t$ Test & $t$ Test & Correl. & $t$ Test & $t$ Test & $t$ Test & $t$ Test \\
\hline AURKB & 0.003 & 0.64 & 0.65 & 0.25 & $2 \mathrm{E}-55$ & $5 E-42$ & 0.057 & $2 E-36$ \\
\hline DNMTS & 0.061 & 0.64 & 0.90 & 0.052 & $7 \mathrm{E}-50$ & $4 \mathrm{E}-41$ & 1E-04 & $9 \mathrm{E}-24$ \\
\hline$E Z H 2$ & 0.16 & 0.64 & 0.90 & 0.48 & $5 E-48$ & $1 \mathrm{E}-31$ & 0.057 & $9 \mathrm{E}-34$ \\
\hline SUV39H1 & 0.008 & 0.78 & 0.78 & 0.25 & $1 \mathrm{E}-21$ & $1 \mathrm{E}-13$ & 0.34 & $3 \mathrm{E}-14$ \\
\hline SUV39H2 & 0.31 & 0.21 & 0.90 & 0.86 & $5 E-46$ & $3 E-38$ & 0.12 & $3 \mathrm{E}-30$ \\
\hline $\mathrm{CH}$ & 0 & 0.64 & $0.9 c$ & 70 & 7E-19 & $1 \mathrm{E}-10$ & 0.032 & $2 \mathrm{E}-08$ \\
\hline$K D M 4 B$ & 0.16 & 0.50 & 0.30 & 0.22 & $3 E-98$ & $1 E-66$ & 0.021 & $3 \mathrm{E}-52$ \\
\hline MYST1 & 0.55 & 0.79 & 0.90 & 0.40 & $4 \mathrm{E}-10$ & 1E-09 & 0.006 & $2 \mathrm{E}-04$ \\
\hline PCGF2 & 0.63 & 0.020 & 0.30 & 0.34 & $4 \mathrm{E}-18$ & $5 E-11$ & $5 E-07$ & $7 \mathrm{E}-21$ \\
\hline & .31 & 0.75 & 0.30 & 0.48 & $9 \mathrm{E}-15$ & $1 E-07$ & 0.37 & $3 \mathrm{E}-06$ \\
\hline PRMT8 & 0.63 & 0.83 & 0.78 & 0.43 & 0.008 & $7 \mathrm{E}-04$ & 0.001 & 0.11 \\
\hline $\operatorname{SIN} 3 A$ & 0.12 & 0.50 & 0.65 & 0.48 & $1 \mathrm{E}-20$ & $9 \mathrm{E}-17$ & 0.057 & $5 \mathrm{E}-09$ \\
\hline
\end{tabular}
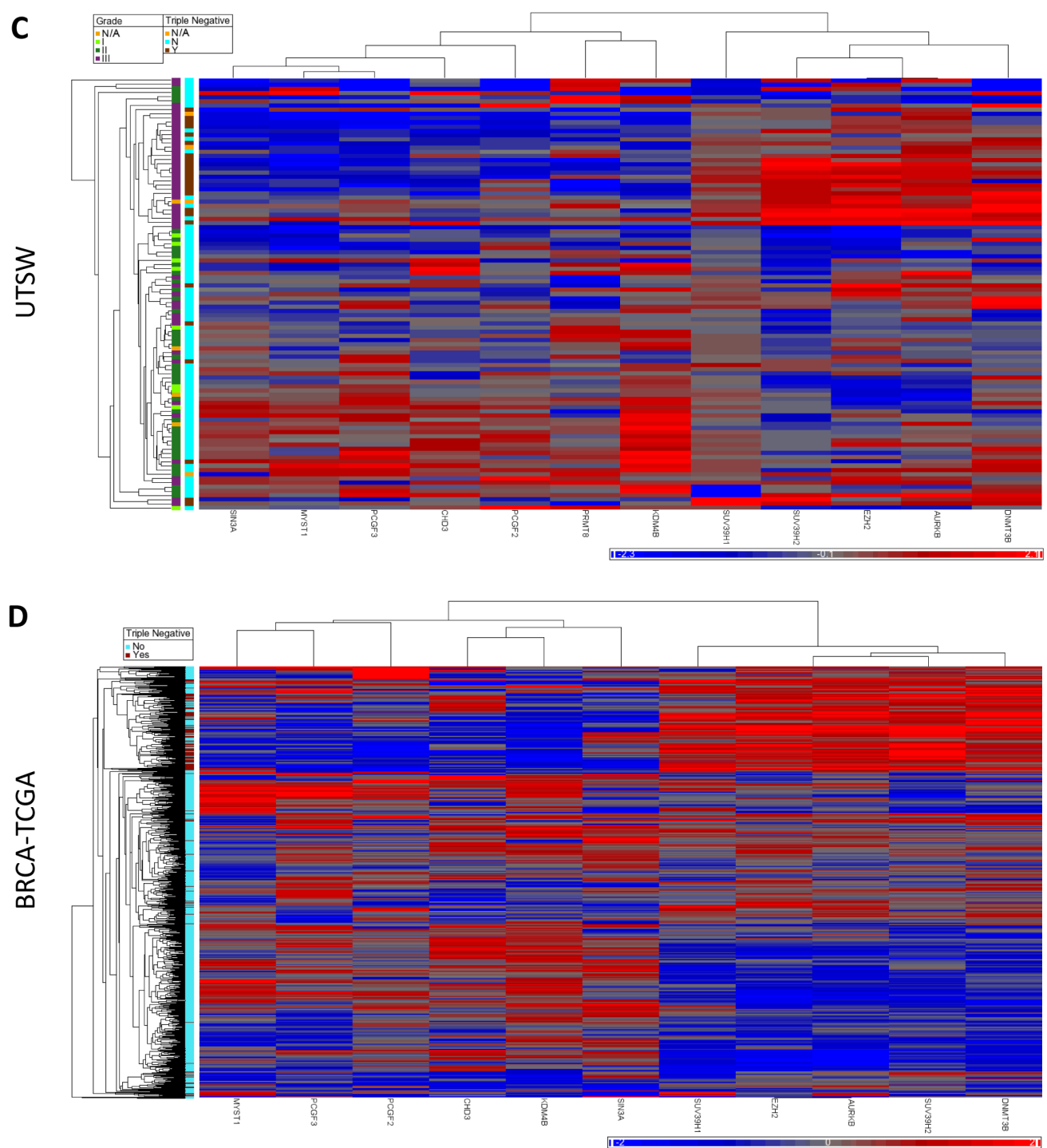

Figure 2: A set of epigenetic modifiers correlates with breast cancer subtypes and defines an Epigenetic Signature associated with tumor grade and triple negative status. A., FDR-corrected $p$ values $(q)$ are shown for genes showing significant associations with at least two clinical variables in our qRT-PCR-based UTSW breast cancer dataset. B., FDR $q$ values were calculated for the RNA-Seq-based BRCA-TCGA dataset analyzed for the genes of the Epigenetic Signature. In A. and B., positive correlations are shown in red and negative correlations in blue. C., Heatmap and unsupervised hierarchical clustering of tumors samples in the UTSW collection ( $n=103$ ) using the expression levels of the 12 genes shown in A. Grade and triple negative (TN) status of the samples is denoted by the color coded legend on the sides. D., Heatmap and unsupervised hierarchical clustering of tumors samples in the BRCA-TCGA collection $(n=730)$ using the expression levels of the genes shown in B. excepting PRMT8 for which too few samples had RNA-Seq data available. Triple negative status of the samples is denoted by the color coded legend on the sides. Grade information was not available in the TCGA portal. In C. and D., relative expression levels are shown in a blue (low) to red (high) scale representing standard deviations away from the average expression of all samples for each gene (grey). See also Supplementary Figures 3 and 4. 
A

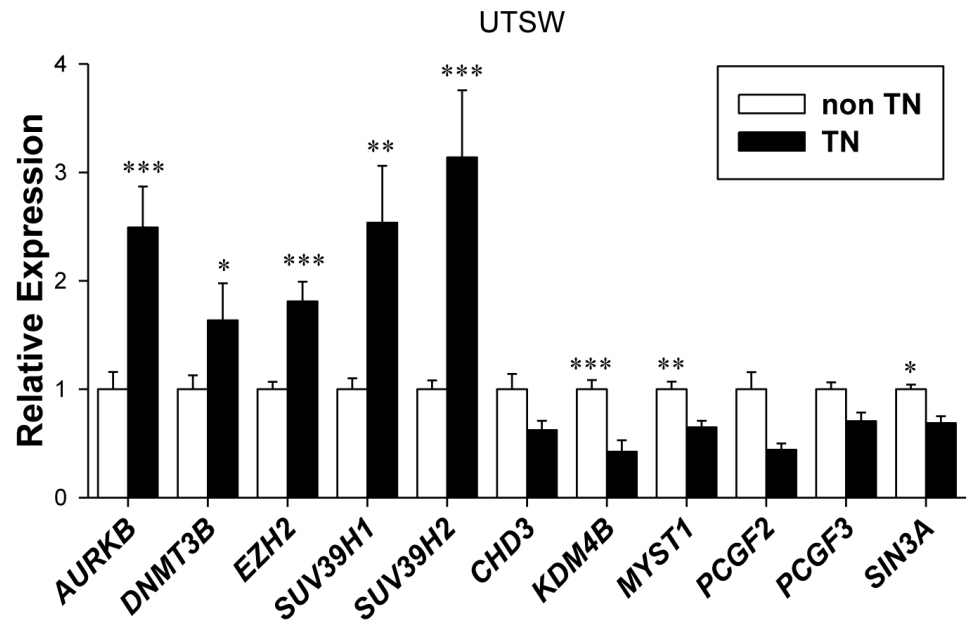

B

TCGA

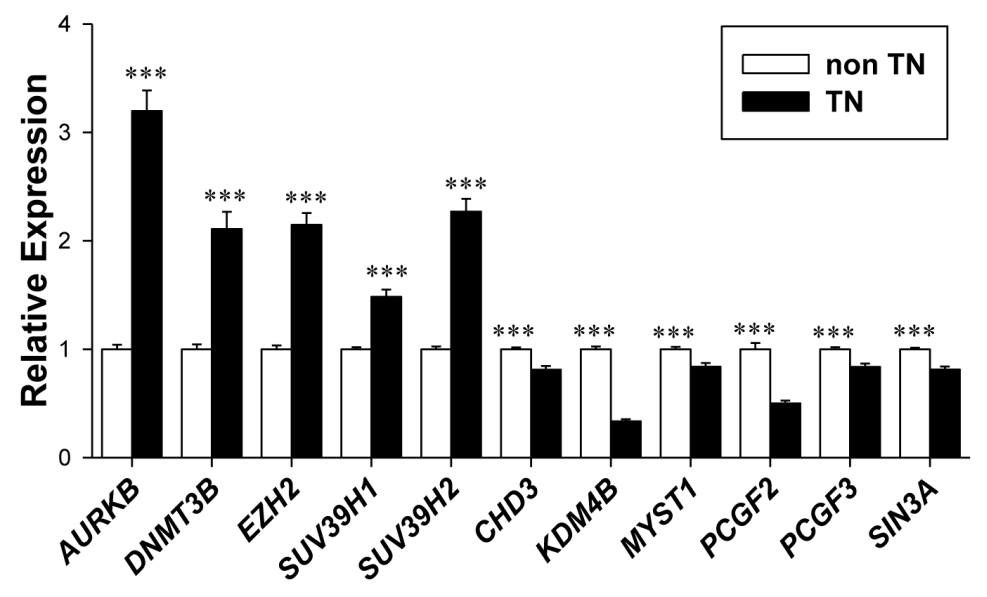

C

METABRIC

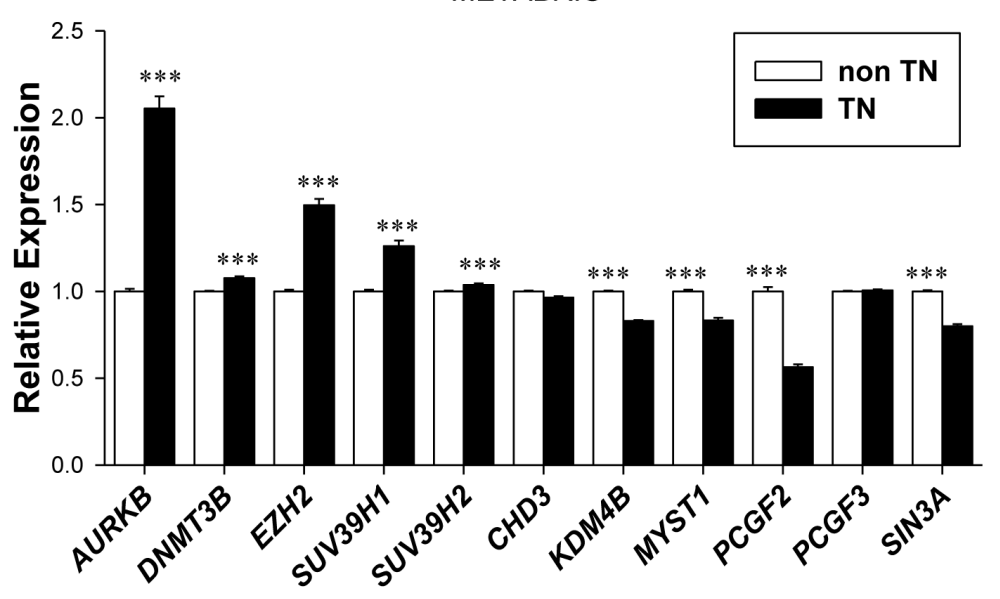

Figure 3: Deregulation of a subset of epigenetic modifiers in Triple Negative (TN) breast cancers across three independent datasets. A., Expression level in the UTSW cohort of the eleven genes from the Epigenetic Signature defined in the UTSW dataset ( $n=25 \mathrm{TN}, n=75$ nonTN) and validated in B. TCGA $(n=120 \mathrm{TN}, n=610$ nonTN) and C. METABRIC ( $n=320 \mathrm{TN}, n=$ 1672 nonTN) datasets. Bars represent mean/average and error bars show standard error. Note that PRMT8 is not included due to paucity of data in TCGA for this gene. 
A

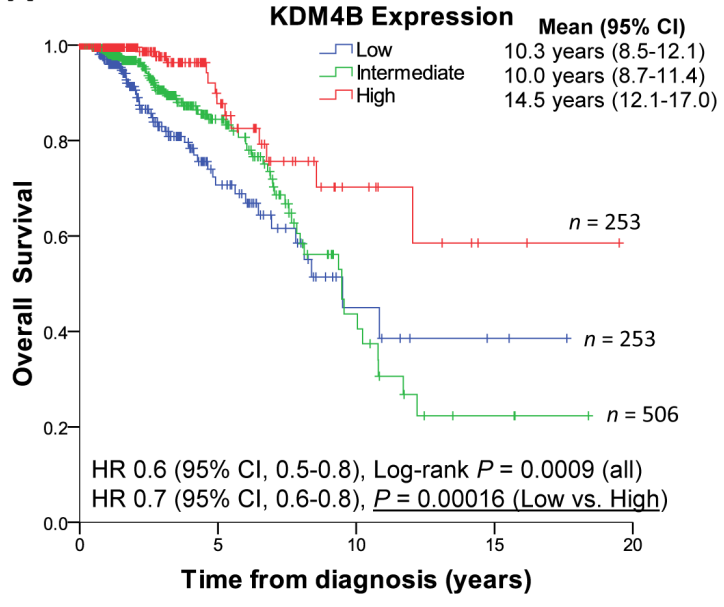

\section{TCGA}

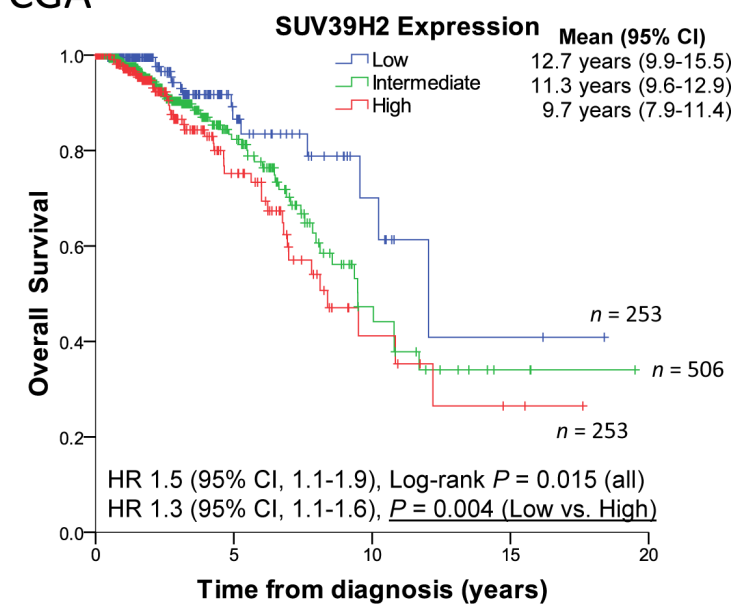

B
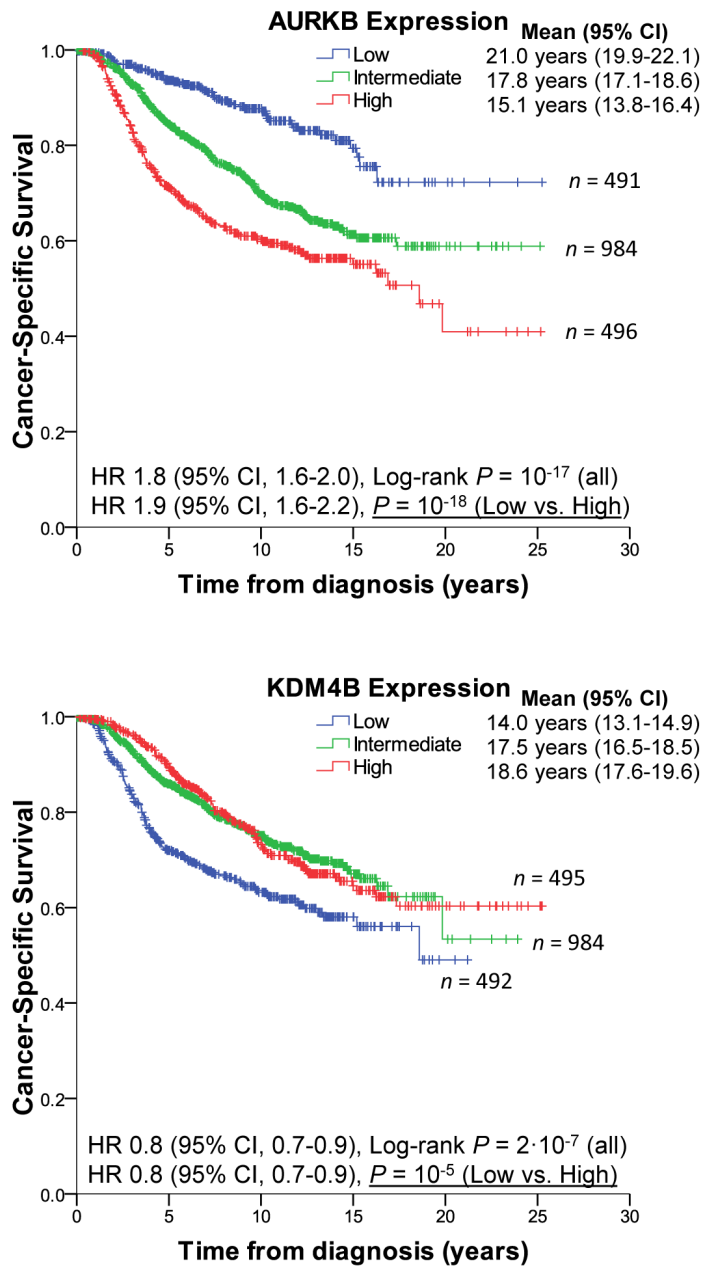

METABRIC
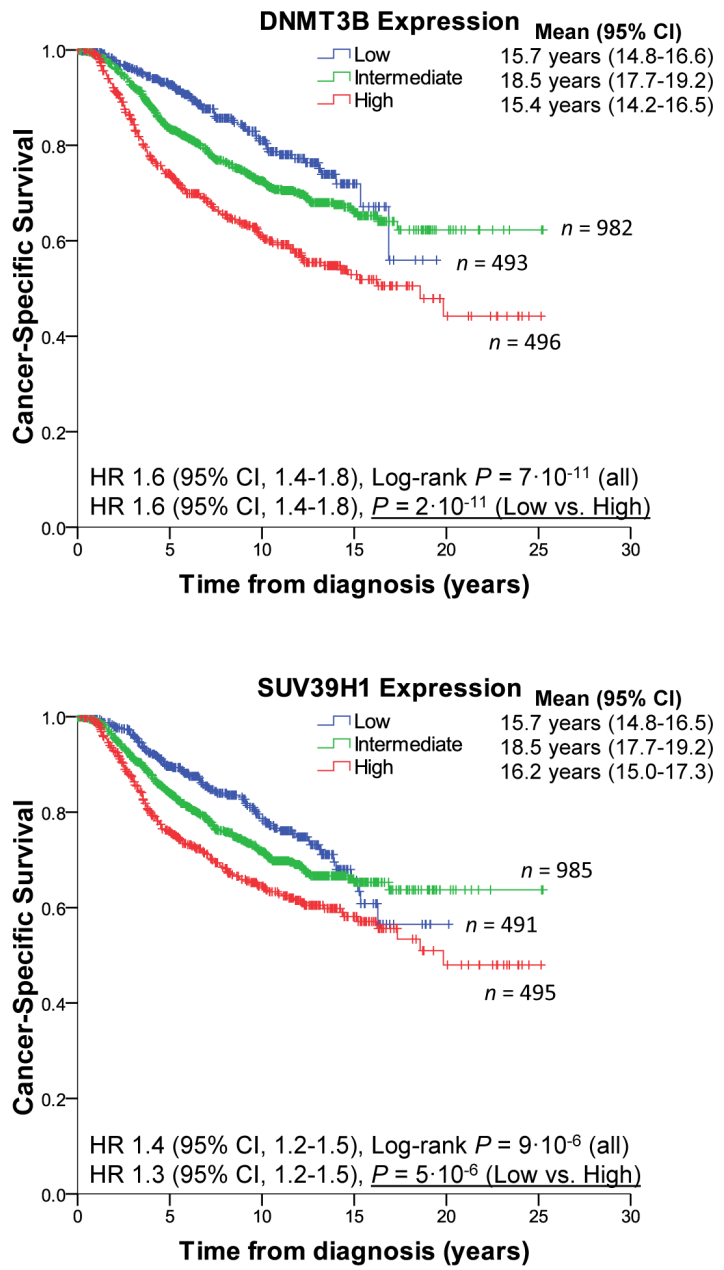

Figure 4: Epigenetic modifier expression levels predict breast cancer patient survival. A., Left: Kaplan-Meier survival curves of BRCA-TCGA patients showing low KDM4B levels ( $1^{\text {st }}$ quartile, blue) are significantly associated with poorer survival than patients with high expression levels ( $4^{\text {th }}$ quartile, red). Second and third quartiles are shown combined, in green. Note that $K D M 4 B$ levels are negatively correlated with tumor grade and triple negative status. Right: BRCA-TCGA patients showing high levels of SUV39H2 ( $4^{\text {th }}$ quartile, red) are significantly associated with poorer survival than patients with low expression levels ( $1^{\text {st }}$ quartile, blue). Note that SUV39H2 levels are positively correlated with triple negative status. B, METABRIC patients expressing high levels of $A U R K B, D N M T 3 B$ or $S U V 39 H 1$ (red curves) or low levels of KDM4B (blue curve) have worse prognosis. Censored cases are designated by crosses. See also Supplementary Figure 5. 
for individual receptor status as shown in Supplementary Tables 3-4.

\section{The epigenetic signature is prognostic of survival}

To evaluate if levels of any of the eleven genes had predictive value, we examined each gene individually in the TCGA cohort, and then validated the results in METABRIC. Strikingly, levels of $K D M 4 B$ on their own had prognostic value in TCGA, with patients harboring high expressing tumors surviving significantly longer (Figure 4A). SUV39H2 levels were also predictive of survival (9.7 yrs. [95\% CI 7.9-11.4 yrs.] for high expressors vs. 12.7 yrs. [95\% CI 9.9-15.5 yrs.] for low expressors; Figure 4A, right). Individually, the other genes in the signature did not segregate poor $v s$. good prognosis in TCGA. Levels of $K D M 4 B$ were predictive of survival also in METABRIC as were levels of $D N M T 3 B$, $S U V 39 H 1$, and as recently described [26], AURKB (Figure 4B). This larger dataset also uncovered significant prognostic value associated with PRMT8 and SIN3A (Supplementary Figure 5) as well as confirmed the wellknown association of high EZH2 expression levels with poor survival in breast cancer.

We next measured the levels of signature genes whose protein products could be pharmacologically targeted in breast cancer lines (AURKB, SUV39H1, $S U V 39 H 2$ and $K D M 4 B)$. KDM4B levels in cell lines correlated with TN status in agreement with patient samples (Figure 5A). We thus tested the KDM/Jumonji inhibitor JIB-04 [27] across a panel of breast cancer lines (Supplementary Figure 6) and found that the line most sensitive to JIB-04 was HCC1419, which is derived from a nonTN grade 2 tumor and expresses high levels of $K D M 4 B$ (Figure 5B). The most resistant line tested of known origin, HCC1937, represents TN grade 3 disease (Figure 5B).

We then evaluated our Epigenetic Signature for prognostic value. We defined the Epigenetic Signature as high risk when the gene expression of at least four of the positive-correlated genes with poor prognosis $(A U R K B$, $D N M T 3 B, E Z H 2, S U V 39 H 1, S U V 39 H 2)$ are in the $4^{\text {th }}$ quartile of the population and/or four of the negativelycorrelated genes (CHD3, KDM4B, MYST1, PCGF2, $P C G F 3, S I N 3 A)$ are in the $1^{\text {st }}$ quartile of the population. This criterion to meet the Epigenetic Signature is not too stringent (just one third of the Epigenetic Signature genes were sufficient to identify patients of high risk), and it gave robust prognostic value in both TCGA and METABRIC (Figure 6A), establishing epigenetic subgroups of clinical significance. Patients with high-risk Epigenetic Signature displayed poor survival in both datasets $(P=0.007$ and $4 \cdot 10^{-12}$, respectively; Figure $\left.6 \mathrm{~A}\right)$, with similar hazard ratios (1.6 and 1.8, respectively). The analysis of patient survival based on TN status (Supplementary Figure 7) showed lower significance in both datasets than the Epigenetic Signature (Figure 6A), indicating that the Epigenetic Signature is a stronger predictor of survival.

To further investigate the prognosis potential of the Epigenetic Signature, we performed univariate Cox regression models on the clinical variables (Table 1).
A

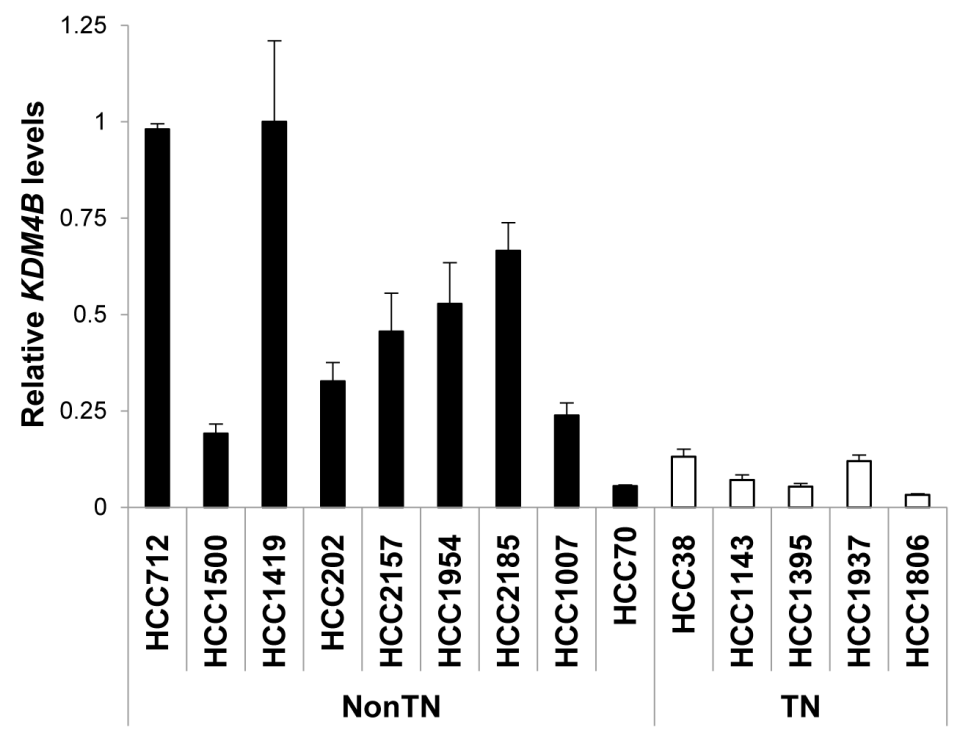

B

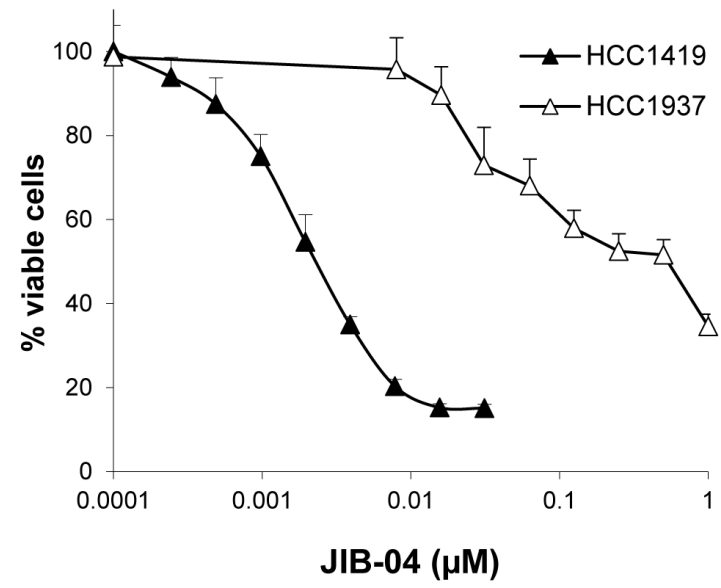

Figure 5: Epigenetic Signature gene $K D M 4 B$ is targetable in nonTN grade 2 breast cancer cells. A., Levels of $K D M 4 B$ measured by qRT-PCR across a panel of breast cancer cell lines based on the triple negative (TN) status is shown. Error bars represent standard deviations across triplicates. B., Response of nonTN, grade 2 HCC1419 cells (high KDM4B) vs. TN, grade 3 HCC1937 cells (low $K D M 4 B$ ) to Jumonji inhibitor JIB-04. See also Supplementary Figure 6. 
A

Epigenetic Signat
At least any of 4 events:
High Gene Expression
(4th quartile) of:
AURKB
DNMT3B
EZH2
SUV $39 H 1$
SUV39H2
and/or
Low Gene Expression
(1st quartile) of:
CHD3
KDM4B
MYST1
PCGF2
PCGF3
SIN3A

TCGA

METABRIC
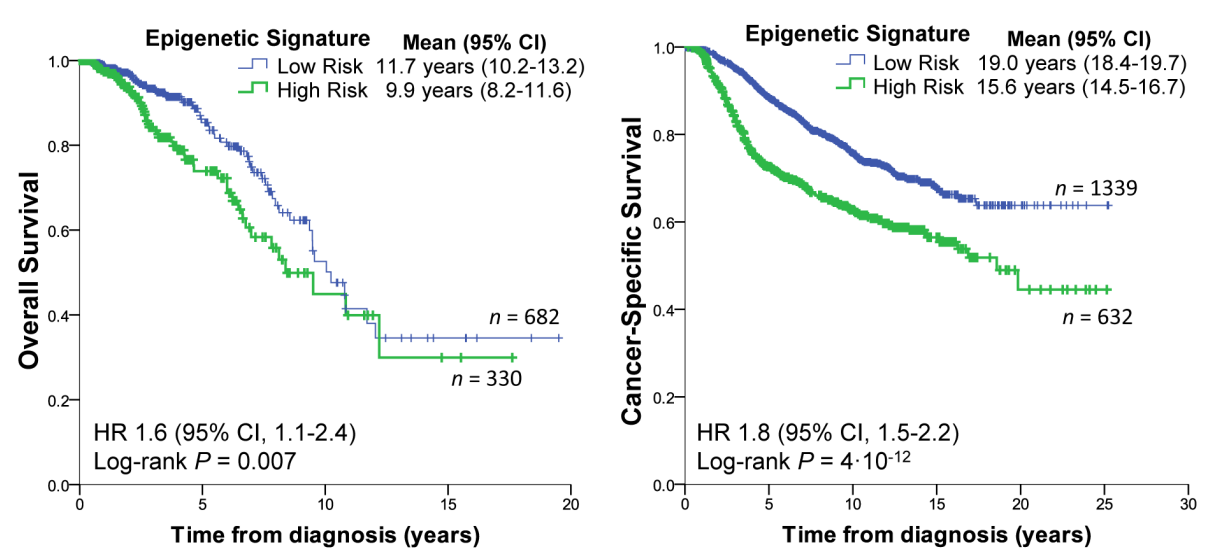

B
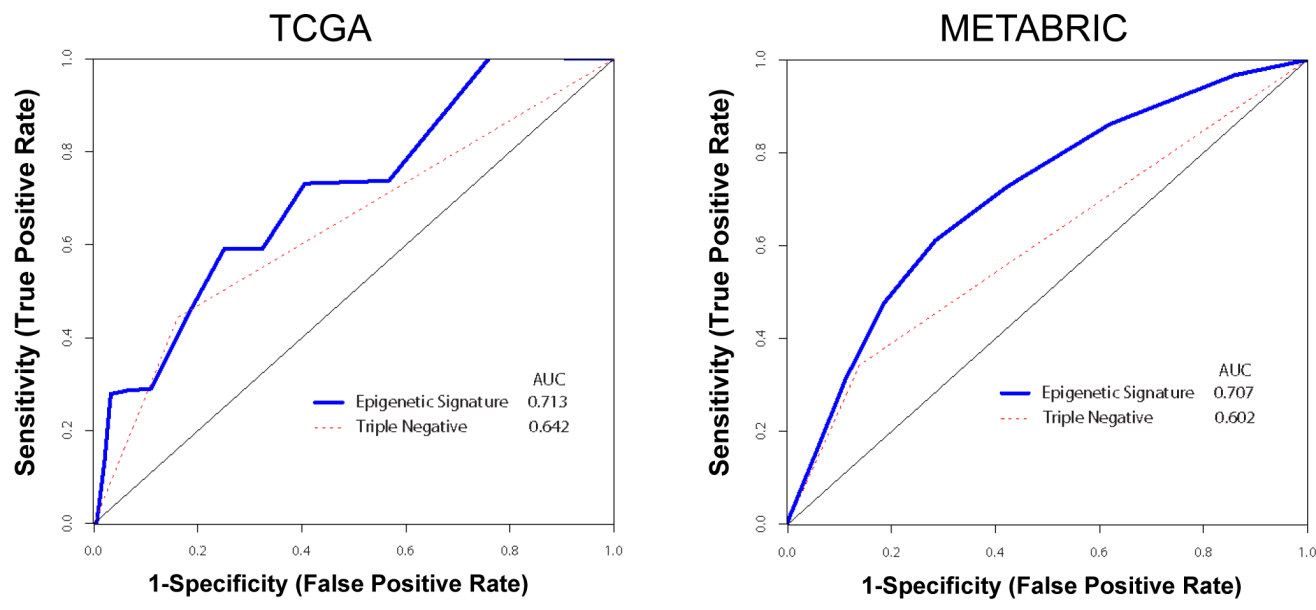

C

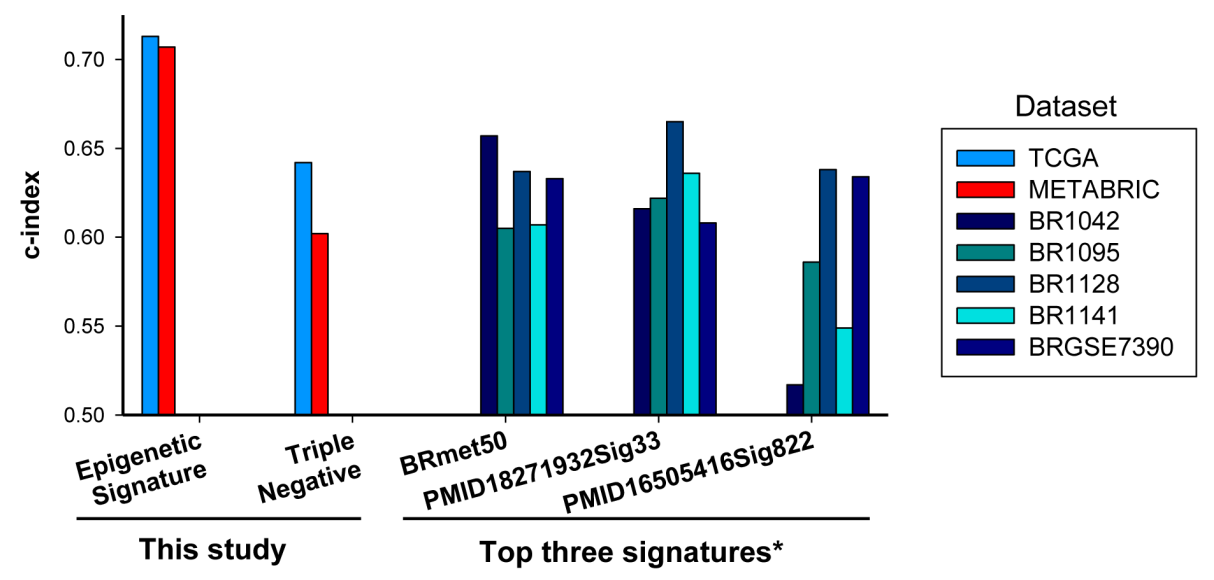

Figure 6: The Epigenetic Signature is a predictor of survival that outperforms other signatures. A., The Epigenetic Signature can identify breast cancer patients with poor survival in both TCGA and METABRIC datasets. The signature is met when at least the expression values of four positively-correlated genes with tumor grade and TN (AURKB, DNMT3B, EZH2, SUV39H1, SUV39H2) are high (in the $4^{\text {th }}$ quartile of the population) and/or the expression values of four negatively-correlated genes with tumor grade and TN (CHD3, KDM4B, MYST1, PCGF2, PCGF3, SIN3A) are low (in the 1st quartile of the population). B., Receiver Operator Characteristic (ROC) curve analysis of the Epigenetic Signature on patient survival using the survivalROC package in R. The area under the curve (AUC) of the Epigenetic Signature is greater than the triple negative status in the TCGA and METABRIC datasets. C., Comparison of the ROC analysis of the Epigenetic Signature and TN status of this study with the data reported by Lehmann and colleagues [28] on the top three signatures in breast cancer. See also Supplementary Figure 7. 
Table 1: Univariate and multivariate Cox regressions of the clinical variables and the Epigenetic Signature in the TCGA and METABRIC datasets.

\begin{tabular}{|l|l|l|l|l|l|l|l|l|l|l|l|l|}
\hline & \multicolumn{4}{|c|}{ TCGA } & \multicolumn{6}{c|}{ METABRIC } \\
\hline & \multicolumn{3}{|c|}{ Univariate Analysis } & Multivariate Analysis & \multicolumn{3}{c|}{ Univariate Analysis } & \multicolumn{2}{c|}{ Multivariate Analysis } \\
\hline \multicolumn{1}{|c|}{ Variable } & HR & $\mathbf{9 5 \%}$ CI & $\boldsymbol{P}$ & HR & $\mathbf{9 5 \%}$ CI & $\boldsymbol{P}$ & HR & $\mathbf{9 5 \%}$ CI & $\boldsymbol{P}$ & HR & $\mathbf{9 5 \%}$ CI & $\boldsymbol{P}$ \\
\hline Age (> median) & 1.6 & $1.1-2.3$ & 0.010 & 2.1 & $1.4-3.1$ & $4 \cdot 10^{-4}$ & 0.9 & $0.7-1.2$ & 0.55 & & & \\
\hline High Grade (3 vs. 1-2) & & & & & & & 1.8 & $1.5-2.2$ & $10^{-10}$ & 1.4 & $1.1-1.8$ & 0.003 \\
\hline High Stage (III-IV vs. I-II) & 2.0 & $1.3-2.9$ & $7 \cdot 10^{-4}$ & 2.2 & $1.5-3.3$ & $7 \cdot 10^{-5}$ & 3.4 & $2.5-4.7$ & $8 \cdot 10^{-15}$ & 3.5 & $2.6-4.8$ & $7 \cdot 10^{-15}$ \\
\hline ER Positive & 0.7 & $0.5-1.1$ & 0.15 & & & & 0.5 & $0.4-0.6$ & $9 \cdot 10^{-12}$ & & & \\
\hline PR Positive & 0.7 & $0.5-1.0$ & 0.042 & & & & 0.5 & $0.4-0.6$ & $2 \cdot 10^{-12}$ & & & \\
\hline HER2 Positive & 1.3 & $0.7-2.4$ & 0.36 & & & & 2.2 & $1.8-2.7$ & $10^{-12}$ & 1.8 & $1.4-2.3$ & $6 \cdot 10^{-6}$ \\
\hline Triple Negative & 1.7 & $0.9-2.9$ & 0.08 & & & & 1.5 & $1.2-1.9$ & $2 \cdot 10^{-4}$ & & & \\
\hline Epigenetic Signature & 1.6 & $1.1-2.4$ & 0.008 & 2.2 & $1.4-3.2$ & $2 \cdot 10^{-4}$ & 1.8 & $1.5-2.2$ & $9 \cdot 10^{-12}$ & 1.5 & $1.2-1.9$ & $4 \cdot 10^{-4}$ \\
\hline
\end{tabular}

HR, Hazard Ratio; CI, Confidence Interval.

Variables that were significant in the univariate Cox model were entered into an unsupervised stepwise forward conditional multivariate analysis to identify independent predictors of prognosis. The Epigenetic Signature was included in the multivariate Cox regression model of both TCGA and METABRIC $\left(P=2 \cdot 10^{-4}\right.$ and $P=4 \cdot 10^{-4}$, respectively, Table 1$)$, while variables that were not completely independent were not present in the final step of the model. Only high stage was a stronger independent prognostic factor in both datasets. Notably, tumor grade was an independent predictor of survival with respect to the Epigenetic Signature in METABRIC. TN status was significant in the univariate Cox model of the METABRIC dataset but excluded from the final multivariate model (Table 1), indicating that its contribution in prognosis is weaker compared to other variables, such as stage or the Epigenetic Signature. A detailed examination of the tumor characteristics of the high-risk Epigenetic Signature population revealed an over-representation of Basal-type and an under-representation of LumB and especially LumA breast cancers (Supplementary Table 5).

To further inspect the prognostic accuracy and diagnostic potential of the Epigenetic Signature, we performed Receiver Operating Characteristic curve analysis (Figure 6B). The Epigenetic Signature showed a greater area under the curve (AUC) than the TN status in both TCGA and METABRIC, with an average prognostic value (c-index) of 0.71 for the Epigenetic Signature and 0.62 for TN status. In addition, the c-index for the Epigenetic Signature was greater than the ones observed for the top 3 gene expression signatures out of 351 reported breast cancer signatures from 206 studies evaluated by Lehmann and colleagues [28], namely BRmet50, PMID18271932Sig33 and PMID16505416Sig822 (Figure 6C). This suggests that the Epigenetic Signature outperforms previous gene expression signatures in the prognosis of breast cancer.

\section{DISCUSSION}

Here we have identified the epigenetic modifiers that become deregulated during human breast oncogenesis. Among these, a set of 11 epigenetic genes distinguish between TN and nonTN human breast cancer specimens and two of these genes independently offer prognostic value. Our results from this novel UTSW dataset were validated in the TCGA and METABRIC datasets confirming the presence of epigenetic subgroups within mammary malignancies and additionally showing the prognostic value of several genes from our Epigenetic Signature, in these larger cohorts. Importantly, our studies reveal that human TN disease may be targetable by inhibitors of EZH2, AURKB, DNMT3B and/or SUV39H1/2 and that nonTN tumors may respond to KDM4B and PRC1 (PCGF2/3) inhibition.

In line with reports that the estrogen receptor (ER) regulates $K D M 4 B$ expression [29], we observed upregulation of $K D M 4 B$ in ER+ but not in ER- or TN tumors in agreement with KDM4B's oncogenic activity in other tumor types [10, 30, 31]. In nonTN tumors we also observed upregulation of members of the PRC1 complex, $P C G F 2$ and $P C G F 3$ [32]. Whether this upregulation of PCGF factors defines a susceptibility to PRC1 inhibitors, such as PRT4165 [33], remains to be investigated.

In addition to the established $E Z H 2$ and the reported AURKB [34-36], three other targetable epigenetic genes were significantly upregulated in TN disease: $D N M T 3 B$, SUV39H1 and SUV39H2. The high expression of $D N M T 3 B$ we report is consistent with the described hypermethylator phenotype of this breast cancer subtype [37] and itself has prognostic value (Figure 4B). Of interest, SUV39H1, shown to negatively regulate the ER promoter [38], is upregulated in TN patient samples and shows prognostic value in the large METABRIC cohort (Figure 4B). This is in agreement with the trend reported by Patani et al. in a smaller patient cohort [39] as is the 
better prognosis of patients with high MYST1/KAT8 (Supplementary Figure 5). The functional significance of this enzyme as well as other epigenetic modifiers in our signature (CHD3, PRMT8, SIN3A) will surely be of future interest [40-42].

Remarkably, we have identified an Epigenetic Signature that is a strong independent predictor of patient survival in the TCGA and METABRIC datasets, according to the multivariate Cox regression models. This Epigenetic Signature outperformed TN status and other clinical variables for prognosis prediction. Notably, ROC analysis revealed a c-index for the Epigenetic Signature that was greater than any of the c-indices observed for the top 3 gene expression signatures out of 351 reported breast cancer signatures from 206 studies evaluated by Lehmann and colleagues [28]. Therefore, the Epigenetic Signature has the potential to be a novel biomarker of patient survival in breast cancer. In summary, we have identified epigenetic breast cancer subgroups overall, and within TN and nonTN human breast cancers, which define novel epigenetic targets and suggest target-combinations for these malignancies.

\section{MATERIALS AND METHODS}

\section{Clinical samples}

The University of Texas Southwestern Medical Center Tissue Resource (UTSTR) was the source of tumor and benign samples from human patients. Samples were processed by the UTSTR after proper consent under IRB approved protocols and the first 103 samples in the collection scored to be $>70 \%$ tumor tissue for cancer samples were used for this study [21] along with matching benign tissue when available. UTSTR de-identified samples and extracted total RNA. Patient and tumor characteristics are described in Supplementary Table 1. A PAM50-like method was used to classify these breast cancers into Luminal A (LumA; tumor grade I or II, ER+, HER2- and Ki67 $\leq 18 \%$ ), Luminal B (LumB; tumor grade III, ER+, HER2- and Ki67 $>18 \%$ or ER + and HER2+), HER2 overexpressing (HER2; ER-, PR- and HER2+) and Basal/Triple Negative (TN; ER-, PR- and HER2-) [43].

\section{qRT-PCR}

Primers sets were designed against the corresponding human genes (sequences are listed in Supplementary Table 2) and validated as previously described [44]. RNA samples from the UTSTR were quantified, DNAse treated and reverse transcribed, and the resulting complementary DNA (cDNA) was amplified in SYBR real-time quantitative PCR assays (Applied
Biosystems) using a high throughput robotic platform. Reactions were performed on an ABI Prism 7900HT with an initial 2-min pre-incubation at $50{ }^{\circ} \mathrm{C}$, followed by 10 min at $95^{\circ} \mathrm{C}$ and then 40 cycles of $95{ }^{\circ} \mathrm{C}$ for $15 \mathrm{sec}$ and $60{ }^{\circ} \mathrm{C}$ for $1 \mathrm{~min}$. hCyclophilin was used as the reference gene and hTBP or $18 \mathrm{~S}$ used as a second reference gene to confirm expression changes. Data were analyzed following the $\Delta \Delta \mathrm{Ct}$ method as described previously [44], using validated cDNA standard curves. Reactions were run in triplicate. Tissue specific genes were run in addition to the genes of interest and their expression patterns used in subsequent analysis to ensure correlations did not correspond to fat or stromal content of patient samples (Supplementary Figure 2). For quantification of $K M D 4 B$ levels in breast cancer cell lines, RNA was extracted from exponentially growing cells and the exact same protocol and analysis was used as above except that reactions were prepared manually rather than with a robot.

\section{Data analysis}

Gene expression data was analyzed as previously described [45]. Briefly, unpaired $t$ tests were performed between tumor and benign samples taking into account the group variances. The gene expression for each gene was associated with clinical variables using Spearman correlations, $t$ tests or Fisher exact tests depending on the characteristics of the variables (continuous or categorical). All calculated $p$ values were corrected using the Benjamini and Hochberg false discovery rate (FDR) method to discard false positives by the fact of performing multiple tests. Unsupervised hierarchical clustering was computed with Partek Genomics Suite v6.6.

\section{TCGA data analysis}

To validate the results, RNA-Seq and clinical data of breast invasive carcinoma (BRCA) was downloaded from The Cancer Genome Atlas (TCGA) data portal (https://tcga-data.nci.nih.gov/tcga) on June 14 ${ }^{\text {th }}, 2014$ and tested for associations as indicated [46]. Gene expression levels were estimated by the RNA-Seq ExpectationMaximization (RSEM) normalization method and analyzed as described above. The median and quartiles for the expression of each gene was calculated for all available patients. Gene expression was considered low for patients with expression values in the $1^{\text {st }}$ quartile, intermediate for the $2^{\text {nd }}$ and $3^{\text {rd }}$ quartiles and high for the $4^{\text {th }}$ quartile. Tumors with negative estrogen receptor (ER) status, progesterone receptor (PR) status, and HER2 status by either IHC or FISH were considered as triple negatives. Tumors with undetermined or not evaluated status for any of ER, PR or HER2 were excluded. Overall survival was calculated from the date of diagnosis to the date on 
which the patient dies from any cause. Patients alive at the end of the study period were censored at the date of last follow-up or the last date the patient was known to be alive, whichever was longer. Kaplan-Meier survival curves, log-rank tests and Cox regression models were calculated with SPSS Statistics 17. The hazard ratio (HR) and the $95 \%$ confidence intervals (CI) were estimated for each variable using univariate Cox regression models. To identify independent predictors of survival, only the significant variables in the univariate Cox regression were entered into the multivariate Cox proportional hazard model using a forward conditional method considering the default stepwise probabilities of 0.05 for entry and 0.10 for removal of covariates from the model.

\section{METABRIC data analysis}

Clinical information from the Molecular Taxonomy of Breast Cancer International Consortium (METABRIC) was obtained from the original publication [20]. ER, PR and HER2 status was considered based on their reported expression. Gene expression data from METABRIC using the Illumina HT-12 platform was downloaded from the European Genome-Phenome Archive (http://www.ebi. ac.uk/ega/) under accession number EGAS00000000083. Gene expression values for probes corresponding to the same gene were pooled. Only cancer-specific survival was considered and analyzed as described above.

\section{ROC analysis}

Receiver Operating Characteristic (ROC) curve analysis for the Epigenetic Signature and triple negative status on patient survival was performed using the survivalROC package in R 3.2.5 as described [47]. For the Epigenetic Signature, the total amount of genes in the $1^{\text {st }}$ and $4^{\text {th }}$ quartile, according to the signature definition (Figure 6C), were taken into account for each patient instead of considering them only as low or high risk. Half of the median survival was considered as the time point of interest. The Area Under the Curve (AUC) was computed with the Kaplan-Meier estimator.

\section{Cell culture and viability assays}

Breast cancer cell lines were the gift of Dr. D. Euhus and Dr. J. Minna and include the following lines established at our institution: HCC712, HCC1500, HCC1419, HCC202, HCC2157, HCC1954, HCC2185, HCC1007, HCC70, HCC38, HCC1143, HCC 1395, HCC1937 and HCC1806. Cell lines were previously characterized as described [48] and were routinely fingerprinted and mycoplasma tested, grown in RPMI media supplemented with $5-10 \%$ fetal bovine serum.
For cell viability assays, cells were plated at low density in 96-well plates and grown overnight, then exposed to increasing doses of drug treatment or vehicle control. Standard MTS viability assays were performed on the $4^{\text {th }}$ day of treatment and $\mathrm{IC}_{50}$ calculated, as described [27]. JIB-04 was synthesized in-house, as previously reported [27].

\section{ACKNOWLEDGMENTS}

We are grateful to Drs. Bookout and Sondhi for assistance with qRT-PCR robotics, to Ewa Borowicz and Kenneth Spence for primer design and validation, and to $\mathrm{M}$. Varghese for technical help. This work was partly funded by the NCI (R01CA12526901 to E.D.M.), by the Friends of the Cancer Center, by The Welch Foundation (I-1878 to E.D.M.; I-1751 to Y.W.), by CPRIT (RP120717, and RP160493 to E.D.M.; RP130145 to Y.W.), and by DOD (W81XWH-13-1-0318 to Y.W.). Research reported in this publication was partly supported by the National Center for Advancing Translational Sciences of the National Institutes of Health under award Number UL1TR001105 to E.D.M. and S.P-L. The content is solely the responsibility of the authors and does not necessarily represent the official views of the NIH. Authors report no financial conflicts of interest.

\section{CONFLICTS OF INTEREST}

$$
\text { No potential conflict of interest to disclose. }
$$

\section{Editorial note}

This paper has been accepted based in part on peerreview conducted by another journal and the authors' response and revisions as well as expedited peer-review in Oncotarget.

\section{REFERENCES}

1. Polyak K. Breast cancer: origins and evolution. The Journal of Clinical Investigation. 2007; 117(11):3155-3163.

2. Huang Y, Greene E, Murray Stewart T, Goodwin AC, Baylin SB, Woster PM and Casero RA, Jr. Inhibition of lysine-specific demethylase 1 by polyamine analogues results in reexpression of aberrantly silenced genes. Proceedings of the National Academy of Sciences of the United States of America. 2007; 104(19):8023-8028.

3. Jones PA and Baylin SB. The epigenomics of cancer. Cell. 2007; 128(4):683-692.

4. Fucito A, Lucchetti C, Giordano A and Romano G. Genetic and epigenetic alterations in breast cancer: what are the perspectives for clinical practice? International Journal of Biochemistry \& Cell Biology. 2008; 40(4):565-575. 
5. Glozak MA, Sengupta N, Zhang X and Seto E. Acetylation and deacetylation of non-histone proteins. Gene. 2005; 363:15-23.

6. Rathert P, Dhayalan A, Murakami M, Zhang X, Tamas R, Jurkowska R, Komatsu Y, Shinkai Y, Cheng X and Jeltsch A. Protein lysine methyltransferase G9a acts on non-histone targets. Nature Chemical Biology. 2008; 4(6):344-346.

7. Van Rechem C, Black JC, Boukhali M, Aryee MJ, Graslund S, Haas W, Benes CH and Whetstine JR. Lysine demethylase KDM4A associates with translation machinery and regulates protein synthesis. Cancer Discovery. 2015; 5(3):255-263.

8. Schneider P, Bayo-Fina JM, Singh R, Kumar Dhanyamraju P, Holz P, Baier A, Fendrich V, Ramaswamy A, Baumeister S, Martinez ED and Lauth M. Identification of a novel actin-dependent signal transducing module allows for the targeted degradation of GLI1. Nature Communications. 2015; 6:8023.

9. Bayo J, Dalvi MP and Martinez ED. Successful strategies in the discovery of small-molecule epigenetic modulators with anticancer potential. Future Medicinal Chemistry. 2015; 7(16):2243-2261.

10. Hojfeldt JW, Agger K and Helin K. Histone lysine demethylases as targets for anticancer therapy. Nature Reviews Drug Discovery. 2013; 12(12):917-930.

11. Xu B, Konze KD, Jin J and Wang GG. Targeting EZH2 and PRC2 dependence as novel anticancer therapy. Experimental Hematology. 2015; 43(8):698-712.

12. Ezponda T and Licht JD. Molecular pathways: deregulation of histone h3 lysine 27 methylation in cancer-different paths, same destination. Clinical Cancer Research. 2014; 20(19):5001-5008.

13. Helming KC, Wang $X$ and Roberts CW. Vulnerabilities of mutant SWI/SNF complexes in cancer. Cancer Cell. 2014; 26(3):309-317.

14. Euhus DM, Bu D, Milchgrub S, Xie XJ, Bian A, Leitch $\mathrm{AM}$ and Lewis CM. DNA methylation in benign breast epithelium in relation to age and breast cancer risk. Cancer Epidemiology, Biomarkers \& Prevention. 2008; 17(5):1051-1059.

15. Simon JA and Lange CA. Roles of the EZH2 histone methyltransferase in cancer epigenetics. Mutation Research. 2008.

16. Sunami E, Shinozaki M, Sim MS, Nguyen SL, Vu AT, Giuliano AE and Hoon DS. Estrogen receptor and HER2/ neu status affect epigenetic differences of tumor-related genes in primary breast tumors. Breast Cancer Research. 2008; 10(3):R46.

17. Kampranis SC and Tsichlis PN. Histone demethylases and cancer. Advances in Cancer Research . 2009; 102:103-169.

18. Sharma SV, Lee DY, Li B, Quinlan MP, Takahashi F, Maheswaran S, McDermott U, Azizian N, Zou L, Fischbach MA, Wong KK, Brandstetter K, Wittner B, Ramaswamy $\mathrm{S}$, Classon $\mathrm{M}$ and Settleman J. A chromatin-mediated reversible drug-tolerant state in cancer cell subpopulations. Cell. 2010; 141(1):69-80.

19. Kaelin WG, Jr. and Thompson CB. Q\&A: Cancer: clues from cell metabolism. Nature. 465(7298):562-564.

20. Curtis C, Shah SP, Chin SF, Turashvili G, Rueda OM, Dunning MJ, Speed D, Lynch AG, Samarajiwa S, Yuan Y, Graf S, Ha G, Haffari G, et al. The genomic and transcriptomic architecture of 2,000 breast tumours reveals novel subgroups. Nature. 2012; 486(7403):346-352.

21. Peña-Llopis $\mathrm{S}$ and Brugarolas J. Simultaneous isolation of high-quality DNA, RNA, miRNA and proteins from tissues for genomic applications. Nature Protocols. 2013; 8(11):2240-2255.

22. Reiche K, Kasack K, Schreiber S, Luders T, Due EU, Naume B, Riis M, Kristensen VN, Horn F, Borresen-Dale AL, Hackermuller J and Baumbusch LO. Long non-coding RNAs differentially expressed between normal versus primary breast tumor tissues disclose converse changes to breast cancer-related protein-coding genes. PLoS ONE. 2014; 9(9):e106076.

23. Kim HS, Vassilopoulos A, Wang RH, Lahusen T, Xiao Z, Xu X, Li C, Veenstra TD, Li B, Yu H, Ji J, Wang XW, Park SH, Cha YI, Gius D and Deng CX. SIRT2 maintains genome integrity and suppresses tumorigenesis through regulating APC/C activity. Cancer Cell. 2011; 20(4):487499.

24. Berdel B, Nieminen K, Soini Y, Tengstrom M, Malinen M, Kosma VM, Palvimo JJ and Mannermaa A. Histone demethylase GASC1 - a potential prognostic and predictive marker in invasive breast cancer. BMC Cancer. 2012; 12:516.

25. Wiegand KC, Shah SP, Al-Agha OM, Zhao Y, Tse K, Zeng T, Senz J, McConechy MK, Anglesio MS, Kalloger SE, Yang W, Heravi-Moussavi A, Giuliany R, et al. ARID1A mutations in endometriosis-associated ovarian carcinomas. The New England Journal of Medicine. 2010; 363(16):1532-1543.

26. Zhang Y, Jiang C, Li H, Lv F, Li X, Qian X, Fu L, Xu $\mathrm{B}$ and Guo X. Elevated Aurora B expression contributes to chemoresistance and poor prognosis in breast cancer. International Journal of Clinical and Experimental Pathology. 2015; 8(1):751-757.

27. Wang L, Chang J, Varghese D, Dellinger M, Kumar S, Best AM, Ruiz J, Bruick R, Peña-Llopis S, Xu J, Babinski DJ, Frantz DE, Brekken RA, et al. A small molecule modulates Jumonji histone demethylase activity and selectively inhibits cancer growth. Nature Communications. 2013; 4:2035.

28. Lehmann BD, Ding Y, Viox DJ, Jiang M, Zheng Y, Liao W, Chen X, Xiang W and Yi Y. Evaluation of public cancer datasets and signatures identifies TP53 mutant signatures with robust prognostic and predictive value. BMCCancer. 2015; 15:179.

29. Yang J, Jubb AM, Pike L, Buffa FM, Turley H, Baban D, 
Leek R, Gatter KC, Ragoussis J and Harris AL. The histone demethylase JMJD2B is regulated by estrogen receptor alpha and hypoxia, and is a key mediator of estrogen induced growth. Cancer Research. 2010; 70(16):6456-6466.

30. Berry WL and Janknecht R. KDM4/JMJD2 histone demethylases: epigenetic regulators in cancer cells. Cancer Research. 2013; 73(10):2936-2942.

31. Duan L, Rai G, Roggero C, Zhang QJ, Wei Q, Ma SH, Zhou Y, Santoyo J, Martinez ED, Xiao G, Raj GV, Jadhav A, et al. KDM4/JMJD2 Histone Demethylase Inhibitors Block Prostate Tumor Growth by Suppressing the Expression of AR and BMYB-Regulated Genes. Chemistry \& Biology. 2015; 22(9):1185-1196.

32. Gao Z, Zhang J, Bonasio R, Strino F, Sawai A, Parisi F, Kluger Y and Reinberg D. PCGF homologs, CBX proteins, and RYBP define functionally distinct PRC1 family complexes. Molecular Cell. 2012; 45(3):344-356.

33. Ismail IH, McDonald D, Strickfaden H, Xu Z and Hendzel MJ. A small molecule inhibitor of polycomb repressive complex 1 inhibits ubiquitin signaling at DNA doublestrand breaks. The Journal of Biological Chemistry. 2013; 288(37):26944-26954.

34. Fujii S, Tokita K, Wada N, Ito K, Yamauchi C, Ito Y and Ochiai A. MEK-ERK pathway regulates EZH2 overexpression in association with aggressive breast cancer subtypes. Oncogene. 2011; 30(39):4118-4128.

35. Kalashnikova EV, Revenko AS, Gemo AT, Andrews NP, Tepper CG, Zou JX, Cardiff RD, Borowsky AD and Chen HW. ANCCA/ATAD2 overexpression identifies breast cancer patients with poor prognosis, acting to drive proliferation and survival of triple-negative cells through control of B-Myb and EZH2. Cancer Research. 2010; 70(22):9402-9412.

36. Prat A, Adamo B, Cheang MC, Anders CK, Carey LA and Perou CM. Molecular characterization of basal-like and non-basal-like triple-negative breast cancer. The Oncologist. 2013; 18(2):123-133.

37. Roll JD, Rivenbark AG, Sandhu R, Parker JS, Jones WD, Carey LA, Livasy CA and Coleman WB. Dysregulation of the epigenome in triple-negative breast cancers: basallike and claudin-low breast cancers express aberrant DNA hypermethylation. Experimental and Molecular Pathology. 2013; 95(3):276-287.

38. Zhou Q, Atadja P and Davidson NE. Histone deacetylase inhibitor LBH589 reactivates silenced estrogen receptor alpha (ER) gene expression without loss of DNA hypermethylation. Cancer Biology \& Therapy . 2007; 6(1):64-69.
39. Patani N, Jiang WG, Newbold RF and Mokbel K. Histonemodifier gene expression profiles are associated with pathological and clinical outcomes in human breast cancer. Anticancer Research. 2011; 31(12):4115-4125.

40. Li W and Mills AA. Architects of the genome: CHD dysfunction in cancer, developmental disorders and neurological syndromes. Epigenomics. 2014; 6(4):381-395.

41. Lee YH and Stallcup MR. Minireview: protein arginine methylation of nonhistone proteins in transcriptional regulation. Molecular Endocrinology. 2009; 23(4):425-433.

42. Kadamb R, Mittal S, Bansal N, Batra H and Saluja D. Sin3: insight into its transcription regulatory functions. European Journal of Cell Biology. 2013; 92(8-9):237-246.

43. Cheang MC, Chia SK, Voduc D, Gao D, Leung S, Snider J, Watson M, Davies S, Bernard PS, Parker JS, Perou CM, Ellis MJ and Nielsen TO. Ki67 index, HER2 status, and prognosis of patients with luminal B breast cancer. Journal of the National Cancer Institute. 2009; 101(10):736-750.

44. Bookout AL, Cummins CL, Mangelsdorf DJ, Pesola JM and Kramer MF. High-throughput real-time quantitative reverse transcription PCR. Current Protocols in Molecular Biology / edited by Frederick M Ausubel et al. 2006; Chapter 15:Unit 1518.

45. Peña-Llopis S, Vega-Rubin-de-Celis S, Schwartz JC, Wolff NC, Tran TA, Zou L, Xie XJ, Corey DR and Brugarolas J. Regulation of TFEB and V-ATPases by mTORC1. The EMBO Journal. 2011; 30(16):3242-3258.

46. Peña-Llopis S, Christie A, Xie XJ and Brugarolas J. Cooperation and antagonism among cancer genes: the renal cancer paradigm. Cancer Research. 2013; 73(14):41734179.

47. Heagerty PJ, Lumley T and Pepe MS. Time-dependent ROC curves for censored survival data and a diagnostic marker. Biometrics. 2000; 56(2):337-344.

48. Gazdar AF, Kurvari V, Virmani A, Gollahon L, Sakaguchi M, Westerfield M, Kodagoda D, Stasny V, Cunningham HT, Wistuba, II, Tomlinson G, Tonk V, Ashfaq R, Leitch AM, Minna JD and Shay JW. Characterization of paired tumor and non-tumor cell lines established from patients with breast cancer. International Journal of Cancer. 1998; 78(6):766-774. 